Pacific Journal of Mathematics

A GRAPH AND ITS COMPLEMENT WITH SPECIFIED
PROPERTIES. VI. CHROMATIC AND ACHROMATIC
NUMBERS

in Akiyama, Frank horary and Phillip Arthur Ostrand 


\title{
A GRAPH AND ITS COMPLEMENT WITH SPECIFIED PROPERTIES VI: CHROMATIC AND ACHROMATIC NUMBERS
}

\author{
Dedicated to Ruth Bari
}

\section{Jin Akiyama, Frank Harary and Phillip Ostrand}

\begin{abstract}
We characterize the graphs $G$ such that both $G$ and its complement $\bar{G}$ are $n$-colorable, and we specify explicitly all 171 graphs for the case $n=3$. We then determine the 41 graphs for which both $G$ and $\bar{G}$ have achromatic number 3 .
\end{abstract}

1. Introduction. We follow the terminology and notation of [1] but we include some basic definitions for completeness. A coloring of a graph $G$ is an assignment of colors to its points so that whenever two points are adjacent they are colored differently. An $n$-coloring of $G$ is a coloring of $G$ which uses $n$ colors. A complete $n$-coloring of $G$ is an $n$-coloring of $G$ such that, for every pair of distinct colors there exists a pair of adjacent points in $G$ which receive the given pair of colors. The chromatic number $\chi=\chi(G)$ of a graph $G$ is the least integer $n$ such that $G$ has an $n$-coloring. We say that $G$ is $n$-colorable if $\chi(G) \leq n$. Alternatively, $\chi(G)$ can be characterized as the least integer $n$ such that $V(G)$ has a partition into $n$ subsets each of which induces a totally disconnected subgraph. Obviously if $n=\chi(G)$ then every $n$-coloring of $G$ is complete. The achromatic number $\psi=\psi(G)$ of a graph $G$ is the greatest integer $m$ such that $G$ has a complete $m$-coloring. Clearly every graph $G$ of order $p$ has a $p$-coloring, but this coloring is only complete if $G$ is $K_{p}$.

A homomorphism of a graph $G$ onto a graph $G^{\prime}$ is a function $\phi$ from $V(G)$ onto $V\left(G^{\prime}\right)$ such that, whenever $u$ and $v$ are adjacent points of $G$, their images $\phi(u)$ and $\phi(v)$ are adjacent in $G^{\prime}$. Since no point of a graph is adjacent with itself, two adjacent points of $G$ cannot have the same image under any homomorphism of $G$. If $G^{\prime}$ is the image of $G$ under a homomorphism $\phi$, we write $G^{\prime}=\phi(G)$. The order of $\phi$ is $|V(\phi(G))|$. A homomorphism $\phi$ of $G$ is complete of order $n$ if $\phi(G)=K_{n}$. Thus every graph $G$ has a complete homomorphism of order $\chi(G)$ and also a complete homomorphism of order $\psi(G)$, and $\chi(G)$ and $\psi(G)$ are the smallest and largest orders of the complete homomorphisms of $G$. It was shown by Harary, Hedetniemi and Prins [2] that $G$ also has a complete homomorphism of order $n$ for all intermediate $n$.

It is convenient to write $G>H$ when $H$ is an induced subgraph of $G$. If $X$ is a set of points in a graph $G$ then we use $\langle X\rangle$ to denote the 
subgraph $G$ induced by $X$. If necessary to avoid ambiguity we can write $\langle X\rangle_{G}$ and $\langle X\rangle_{H}$ if $X$ is a set of points in two different graphs $G$ and $H$. We write $\bar{\chi}(G)$ for $\chi(\bar{G})$ and $\bar{\psi}(G)$ for $\psi(\bar{G})$.

2. The chromatic number. We are concerned in this section with those graphs $G$ for which both $G$ and $\bar{G}$ are $n$-colorable.

THEOREM 1. Let $G_{1}, G_{2}, \ldots, G_{k}$ be the components of a graph $G$. Then $\bar{\chi}(G)=\Sigma \bar{\chi}\left(G_{\imath}\right)$.

Proof. We first prove the inequality $\chi(G) \leq \Sigma \chi\left(G_{t}\right)$ holds if $G_{1}, G_{2}, \ldots, G_{k}$ are induced subgraphs of $G$ such that $V(G)=\cup V\left(G_{i}\right)$. For each $1 \leq i \leq k$ there exists a family $\mathbf{S}_{l}$ of subsets $V\left(G_{l}\right)$, whose union is $V\left(G_{i}\right)$, with $\left|\mathbf{S}_{i}\right|=\chi\left(G_{i}\right)$, and such that each $S \in \mathbf{S}_{i}$ induces in $G_{\imath}$ a totally disconnected subgraph. Let $\mathbf{S}=\cup \mathbf{S}_{i}$. Then $\mathbf{S}$ is a family of subsets of $V(G)$, whose union is $V(G)$, such that each $S \in \mathbf{S}$ induces in $G$ a totally disconnected subgraph. Thus $\chi(G) \leq|\mathbf{S}| \leq \sum\left|S_{i}\right|=\sum \chi\left(G_{i}\right)$.

Next we show that $\bar{\chi}(G) \geq \sum \bar{\chi}\left(G_{i}\right)$ if $G_{1}, G_{2}, \ldots, G_{k}$ are the components of $G$. There exists a family $\mathbf{S}$ of subsets of $V(G)$, whose union is $V(G)$, with $|\mathbf{S}|=\bar{\chi}(G)$, such that each $S \in \mathbf{S}$ induces in $\bar{G}$ a totally disconnected subgraph. For each $1 \leq i \leq k$, let $\mathbf{S}_{i}=\left\{S \in \mathbf{S} \mid S \cap V\left(G_{i}\right)\right.$ $\neq \varnothing\}$. Points from different components of $G$ are adjacent in $\bar{G}$, so the subfamilies $\mathbf{S}_{i}, 1 \leq i \leq k$, constitute a partition of $\mathbf{S}$. Each $\mathbf{S}_{i}$ is such that every $S \in \mathbf{S}_{i}$ induces in $\bar{G}_{i}$ a totally disconnected subgraph, so $\left|\mathbf{S}_{i}\right| \geq \bar{\chi}\left(G_{i}\right)$. Thus $\bar{\chi}(G)=|\mathbf{S}|=\Sigma\left|\mathbf{S}_{i}\right| \geq \Sigma \bar{\chi}\left(G_{i}\right)$.

Since each $\bar{G}_{i}$ is an induced subgraph of $\bar{G}$, the theorem is an immediate consequence of the discussion above.

The corollaries which follow include a characterization of graphs $G$ such that $G$ and $\bar{G}$ are both $n$-colorable.

Corollary la. Let $G_{1}, G_{2}, \ldots, G_{k}$ be the components of $G$. Then $G$ and $\bar{G}$ are both $n$-colorable if and only if

(i) $\chi\left(G_{i}\right) \leq n$ for every $1 \leq i \leq k$, and

(ii) $\Sigma \bar{\chi}\left(G_{i}\right) \leq n$.

Proof. This follows directly from Theorem 1 and the fact that $\chi(G)=$ $\max \chi\left(G_{l}\right)$.

COROLLARY 1b. If $G$ has $k$ components, then $\bar{\chi}(G) \geq k$. If $k=\bar{\chi}(G)$, then each component of $G$ is complete.

Proof. As $G$ has $k$ components $G_{i}, \bar{G}$ must contain $K_{k}$. If $k=\bar{\chi}(G)$, then $\Sigma \bar{\chi}\left(G_{i}\right)=k$, so for each $i, \bar{\chi}\left(G_{i}\right)=1$, whence $\bar{G}_{i}$ is totally disconnected and therefore $G_{i}$ is complete. 
For the special case of disconnected graphs $G$ such that $G$ and $\bar{G}$ are both 3-colorable, Theorem 1 leads to a particularly simple characterization.

COROLlaRY 1c. If a graph $G$ is disconnected then $G$ and $\bar{G}$ are both 3-colorable if and only if one of the following conditions is satisfied.

(i) $G$ has exactly 3 components each of which is a complete graph of order no greater than 3.

(ii) $G$ has exactly 2 components, $G_{1}$ and $G_{2}$, and $G_{1}$ is a complete graph of order no greater than 3 , and $G_{2}$ is 3-colorable and $\bar{G}_{2}$ is 2-colorable.

Proof. Let $G_{1}, G_{2}, \ldots, G_{k}$ be the components of a disconnected graph $G$.

Suppose first that $G$ and $\bar{G}$ are both 3-colorable. By Corollary lb we need consider only two possible values of $k$.

Case $1 . k=3$.

In this case $k=\bar{\chi}(G)$ so Corollary $1 \mathrm{~b}$ applies and each $G_{i}$ is complete. Then $\chi(G) \leq 3$ implies that each $G_{i}$ is of order no greater than 3 . In this case $G$ satisfies condition (i).

Case 2. $k=2$.

From Theorem 1 we get $\bar{\chi}\left(G_{1}\right)+\bar{\chi}\left(G_{2}\right)=\bar{\chi}(G) \leq 3$. Without loss of generality we may conclude that $\bar{\chi}\left(G_{1}\right)=1$ and $\bar{\chi}\left(G_{2}\right) \leq 2$. As in Case 1 it follows that $G_{1}$ is complete of order no greater than 3 . Thus $G_{2}$, being a subgraph of $G$, is 3-colorable, and $\bar{G}_{2}$ is 2-colorable because $\bar{\chi}\left(G_{2}\right) \leq 2$. In this case $G$ satisfies condition (ii).

Suppose conversely that $G$ satisfies either (i) or (ii).

Case $1^{\prime}$. $G$ satisfies (i).

Let $G_{1}, G_{2}$ and $G_{3}$ be the components of $G$. Then each $G_{i}$ is complete so $V\left(G_{i}\right)$ induces in $\bar{G}$ a totally disconnected subgraph, thus $\bar{\chi}(G) \leq 3$. Because each $G_{l}$ is of order no greater than 3 we can partition $V(G)$ into three subsets $V_{1}^{\prime}, V_{2}^{\prime}$ and $V_{3}^{\prime}$ such that $\left|V_{i}^{\prime} \cap V\left(\dot{G}_{j}\right)\right| \leq 1$ for $1 \leq j, j \leq 3$. Then each $V_{l}^{\prime}$ induces in $G$ a totally disconnected subgraph, so $\chi(G) \leq 3$. In this case $G$ and $\bar{G}$ are both 3-colorable.

Case 2'. $G$ satisfies (ii).

In this case Corollary la clearly implies that $G$ and $\bar{G}$ are both 3-colorable.

THEOREM 2. If a graph $G$ is $n$-colorable, then $\bar{\chi}(G)$ is the least integer $t$ such that $V(G)$ can be partitioned into $t$ subsets $V_{1}, V_{2}, \ldots, V_{t}$ and for each $1 \leq i \leq t,\left|V_{i}\right| \leq n$ and $V_{i}$ induces a complete subgraph. 
Proof. By definition $\bar{\chi}(G)$ is the least integer $t$ such that $V(\underline{G})$ can be partitioned into $t$ subsets $V_{1}, V_{2}, \ldots, V_{t}$ each of which induces in $\bar{G}$ a totally disconnected subgraph. Also for any subset $S$ of $V(G), S$ induces in $\bar{G}$ a totally disconnected subgraph if and only if $S$ induces in $G$ a complete subgraph, in which case $|S| \leq \chi(G) \leq n$.

The corollaries which follow include another characterization of graphs $G$ such that $G$ and $\bar{G}$ are both $n$-colorable which can usefully be applied to connected graphs.

Corollary 2a. A graph $G$ and its complement are both $n$-colorable if and only if there exist positive integers $s, t \leq n$ such that

For each $1 \leq i \leq s$ there is a positive integer $a_{t} \leq t$ such that $\cup K_{a_{i}}$ is a spanning subgraph of $\bar{G}$.

(ii) For each $1 \leq i \leq t$ there is a positive integer $b_{i} \leq s$ such that $\cup K_{b_{t}}$ is a spanning subgraph of $G$.

Moreover the minimum values of $s$ and $t$ which satisfy these conditions are $\chi(G)$ and $\bar{\chi}(G)$ respectively.

Proof. Suppose first that $G$ and $\bar{G}$ are both $n$-colorable. Let $s=\chi(G)$ and $t=\bar{\chi}(G)$, so $s, t \leq n$. As $G$ is $s$-colorable, by Theorem 2 there is a partition of $V(G)$ into $t=\bar{\chi}(G)$ subsets $V_{1}, \ldots, V_{t}$ such that for each $1 \leq i \leq t,\left|V_{i}\right| \leq s$ and $V_{i}$ induces a complete subgraph in $G$. Writing $b_{i}=\left|V_{i}\right|$, we have $\cup K_{b_{i}}=\cup\left\langle V_{i}\right\rangle$ as a spanning subgraph of $G$.

Similarly, since $\bar{G}$ is $t$-colorable and $\bar{\chi}(G)=s$, the same argument applied to $\bar{G}$ yields $\cup K_{a_{t}}$ as a spanning subgraph of $\bar{G}$ for some sequence of positive integers $a_{i} \leq t$.

Now suppose conversely that $G$ is a graph which satisfies conditions (i) and (ii). By condition (i), there is a partition of $V(G)$ into $s$ subsets $V_{1}, \ldots, V_{s}$ such that for each $1 \leq i \leq s, V_{l}$ induces a complete subgraph in $\bar{G}$. Then each $V_{i}$ induces in $G$ a totally disconnected subgraph. Thus $\chi(G) \leq s \leq n$, so $G$ is $n$-colorable. Also note that the least value of $s$ which can satisfy (i) is $\chi(G)$ since $\chi(G) \leq s$. Similarly by (ii) we deduce $\bar{\chi}(G) \leq t \leq n$, so $\bar{G}$ is $n$-colorable and $\bar{\chi}(G)$ is the minimum possible value for $t$.

COROLlaRY 2 b. If a graph $G$ and its complement are both $n$-colorable then the order of $G$ is at most $n^{2}$.

Although this corollary is clearly a consequence of the partition described in Theorem 2, we should also point out that it is also a special case of the well known result of Nordhaus and Gaddum [3] that the order $p$ of a graph satisfies the inequality, $p \leq \chi \bar{\chi}$. It is convenient to include here another useful consequence of the Nordhaus-Gaddum theorem. 
COROLlaRY 2c. If a graph $G$ and its complement are both $n$-colorable and the order of $G$ exceeds $n(n-1)$, then $\chi(G)=\bar{\chi}(G)=n$.

Proof. Since $\chi(G) \leq n$ and $\bar{\chi}(G) \leq n$, if either were actually less than $n$ then $\chi(G) \cdot \bar{\chi}(G)$ would be no greater than $n(n-1)$.

Our final corollary of this theorem deals again with the special case $n=3$.

COROLlaRY $2 \mathrm{~d}$. If a graph $G$ of order $p$ and its complement $\bar{G}$ are both 3-colorable, then $p \leq 9$ and

(i) if $p=9$, then $G$ and $\bar{G}$ each contain $3 K_{3}$ as a subgraph,

(ii) if $p=8$, then $G$ and $\bar{G}$ each contain $2 K_{3} \cup K_{2}$ as a subgraph,

(iii) if $p=7$, then $G$ and $\bar{G}$ each contain either $K_{3} \cup 2 K_{2}$ or $2 K_{3} \cup K_{1}$ as a subgraph.

Proof. Suppose that $G$ and $\bar{G}$ are both 3-colorable. Then by Corollary $2 \mathrm{~b}$ the order $p$ of $G$ is at most 9 . If $p \geq 7$ then by Lemma 2c, $\chi(G)=$ $\bar{\chi}(G)=3$. Thus by Corollary $2 \mathrm{a}$, depending on the value of $p, G$ and $\bar{G}$ must contain the subgraphs described above.

We complete this section by cataloguing all graphs $G$ of order 6 or less and all disconnected graphs $G$ of order 7,8 or 9 for which $G$ and $\bar{G}$ are both 3-colorable. Because there are 171 graphs in this category we will not illustrate them. Rather we describe each such graph by specifying an ordered triple $(p, q, n)$ where $p$ denotes the order and $q$ the size of the graph and $n$ denotes its numerical designation in the Graph Diagrams in Appendix I of [1]. Every graph of order 6 or less appears in these diagrams and the triple $(p, q, n)$ completely describes such graphs. The disconnected graphs of order 7,8, and 9 for which $\chi \leq 3$ and $\bar{\chi} \leq 3$ do not appear in the diagrams, but their components do, and we indicate such graphs by specifying their components. There are pairs $(p, q)$ for which only one graph of order $p$ and size $q$ exists. Such graphs do not have a numerical designation in the Graph Diagrams. We hereby confer the designation 1 on all such graphs. Thus in the lists which follow the triple $(2,1,1)$ represents the unique graph of order 2 and size 1 , namely $K_{2}$. Our list of disconnected graphs of order 7 through 9 with $\chi=\bar{\chi}=3$ are really complete, by the following argument. By Corollary 1c, all such graphs have 3 components each of order 3 or less or 2 components, $G_{1}$ and $G_{2}$, with $G_{1}$ complete of order 3 or less and $\chi\left(G_{2}\right) \leq 3, \bar{\chi}\left(G_{2}\right) \leq 2$. By the Nordhaus-Gaddum theorem we conclude that the order of $G_{2}$ is no greater than 6 , so $G_{2}$ is in List C, our list of all graphs of order 6 or less with $\chi=3, \bar{\chi}=2$. 
List A. $\chi+\bar{\chi} \leq 4$.

$\chi=\bar{\chi}=1:(1,0,1)$ which is $K_{1}$.

$\chi=1$ and $\bar{\chi}=2:(2,0,1)$ which is $\bar{K}_{2}$.

$\chi=2$ and $\bar{\chi}=1:(2,1,1)$ which is $K_{2}$.

$\chi=1$ and $\bar{\chi}=3:(3,0,1)$ which is $\bar{K}_{3}$.

$\chi=3$ and $\bar{\chi}=1:(3,3,1)$ which is $K_{3}$.

$\chi=\bar{\chi}=2$, connected: $(3,2,1),(4,3,2)$, and $(4,4,2)$ which are $P_{3}, P_{4}$ and $C_{4}$.

$\chi=\bar{\chi}=2$, disconnected: $(3,1,1)$ and $(4,2,2)$ which are $K_{1} \cup K_{2}$ and $2 K_{2}$.

List B. $\chi=2$ and $\bar{\chi}=3$.

Connected: $(4,3,3),(5,4,4),(5,4,6),(5,5,3),(5,6,5)$ and $p=6$ with $(q, n)=(5,7),(5,10),(5,14),(6,7),(6,9),(6,11),(7,5),(7,14),(8,23)$, $(9,17)$.

Disconnected: $(4,1,1),(4,2,1),(5,2,2),(5,3,1),(5,3,4),(5,4,1),(6,3,5)$, and $(6,4,8)$.

List C. $\chi=3$ and $\bar{\chi}=2$.

Connected: $(4,4,1),(4,5,1),(5,5,4),(5,6,1),(5,6,4),(5,6,6),(5,7,1)$, $(5,8,2)$, and $p=6$ with $(q, n)=(7,23),(8,5),(8,14),(9,7),(9,9),(9,11)$, $(10,7),(10,10),(10,14),(11,8),(12,5)$.

Disconnected: $(4,3,1),(5,4,5)$ and $(6,6,17)$.

List D. $\chi=\bar{\chi}=3$, order 6 or less.

Connected: $p=5$ with $(q, n)=(5,2),(5,5),(5,6),(6,2),(7,2) ;(6,5,3)$;

$(p, q)=(6,6)$ with $n=8,10,13,14,18,20$;

$(p, q)=(6,7)$ with $n=6,7,8,9,10,11,12,13,16,19,20,21,24$;

$(p, q)=(6,8)$ with $n=1,2,6,7,8,9,10,11,12,13,16,19,20,21,24$;

$(p, q)=(6,9)$ with $n=2,3,5,8,10,13,14,18,19,20 ;(6,10,3)$, $(6,10,12),(6,10,15)$.

Disconnected: $(5,3,2),(5,4,2),(5,5,1)$;

$p=6$ with $(q, n)=(4,6),(5,12),(5,15),(6,2),(6,3),(6,5),(6,19),(7,1)$, $(7,2)$.

List E. $\chi=\bar{\chi}=3$, of order 7,8 , or 9, disconnected $3 K_{3}, 2 K_{3} \cup$ $K_{2}, K_{3} \cup 2 K_{2}, 2 K_{3} \cup K_{1}$, and $K_{3} \cup G$ where $G$ is any connected graph in List $C$, and $K_{2} \cup G$ where $G$ is any connected graph of order 5 or 6 in List $\mathrm{C}$, and $K_{1} \cup G$ where $G$ is any connected graph of order 6 in List C.

Of the 171 graphs which appear in these lists, 116 have $\chi=\bar{\chi}=3$. In addition to these the complements of the 51 graphs in List $E$ are connected graphs of order 7 through 9 with $\chi=\bar{\chi}=3$. And Corollary $2 \mathrm{~d}$ implies that there are many other graphs of order 7 through 9 with 
$\chi=\bar{\chi}=3$ which are not in our lists, of which one example is $G=C_{7}+e$ where the edge $e$ joins two points whose distance in $C_{7}$ is 2 . In this case clearly both $G$ and $\bar{G}$ contain $K_{3} \cup 2 K_{2}$ as a subgraph so $\chi(G)=\bar{\chi}(G)=$ 3.

3. The achromatic number. We first characterize graphs $G$ with $\psi(G)=2$.

THEOREM 3. A graph $G$ has achromatic number 2 if and only if each component of $G$ is complete bipartite.

Proof. Obviously the union of complete bipartite graphs has $\psi=2$. For the converse, assume that $\psi=2$, then $\chi \leq 2$ since $\chi \leq \psi$ for any graph. Thus $G$ must be bipartite. Moreover each component of $G$ cannot contain $P_{4}$ as an induced subgraph since $\psi\left(P_{4}\right)=3$. Thus each component of $G$ must be complete bipartite.

COROLlaRY 3a. The only graphs with $\psi=\bar{\psi}=2$ are $C_{4}, 2 K_{2}, K_{1,2}$ and $K_{2} \cup K_{1}$.

We now develop some results in the form of five lemmas for finding all graphs with $\psi=\bar{\psi}=3$. We write $u A v$ to indicate adjacency and $u \overline{A v}$ for nonadjacency. The first lemma was proved by exhaustion and we omit the detailed verification.

Lemma 4a. Among all graphs of order 6, only the six graphs $2 K_{3}$, $2 K_{2}+\bar{K}_{2}, C_{4}+\bar{K}_{2}$ and their complements $K_{3,3}, C_{4} \cup K_{2}$ and $3 K_{2}$ satisfy the property that either $G$ or $\bar{G}$ contains two point-disjoint triangles and $\psi=\bar{\psi} \leq 3$.
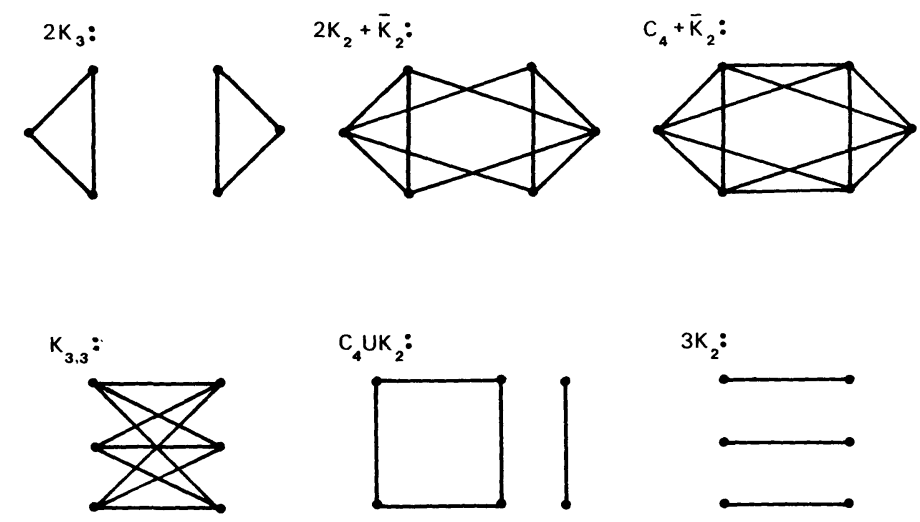

FIGURE 1. The six graphs of order 6 with $\psi, \bar{\psi} \leq 3$ 

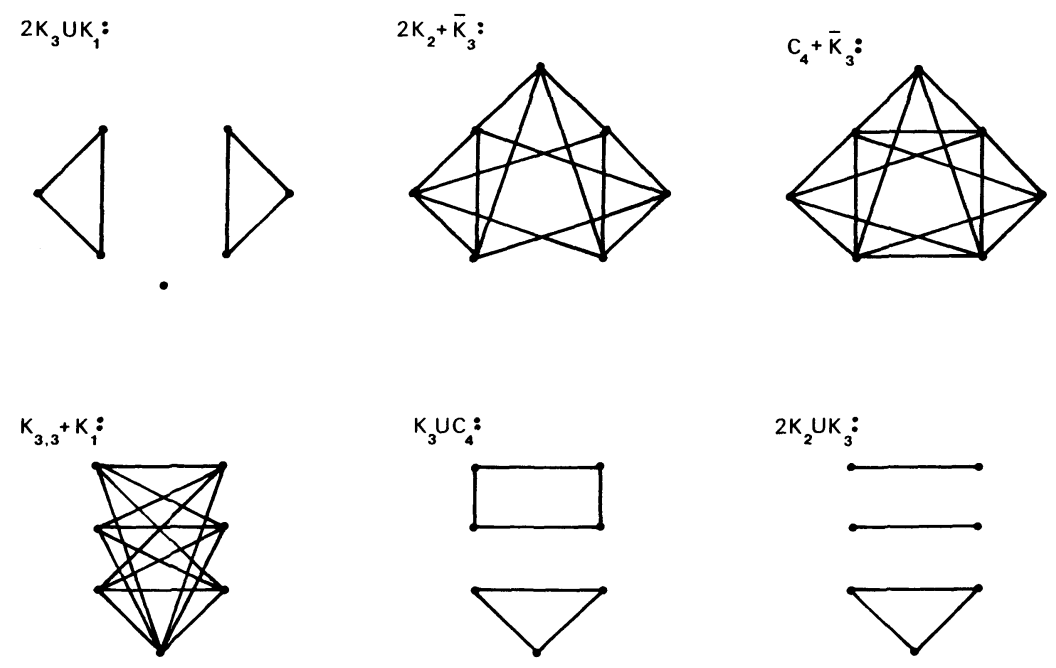

Figure 2. The six graphs of Lemma $4 \mathrm{~b}$

LeMma 4b. Among all graphs of order 7, only the six graphs $2 K_{3} \cup K_{1}$, $2 K_{2}+\bar{K}_{3}, C_{4}+\bar{K}_{3}$ and their complements satisfy the property that either $G$ or $\bar{G}$ contains two point-disjoint triangles and $\psi, \bar{\psi} \leq 3$.

Proof. Assume that $\psi=\bar{\psi}=3$ and that $G$ contains two point-disjoint triangles $T_{1}=\left\{v_{1}, v_{2}, v_{3}\right\}$ and $T_{2}=\left\{v_{4}, v_{5}, v_{6}\right\}$. Then the subgraph $H$ of $G$ induced by these six points in one of the three graphs, $2 K_{3}, K_{2}+\bar{K}_{2}$ or $C_{4}+\bar{K}_{2}$, of Lemma $4 \mathrm{a}$; otherwise either $G$ or $\bar{G}$ contains an induced subgraph of order 6 which has achromatic number at least 4 and so $\psi$ or $\bar{\psi}$ would be at least 4 , a contradiction to the hypothesis. By $w$ we denote the seventh point in $V(G)-V(H)$, and divide the proof into three cases according to whether $H$ is $2 K_{3}, 2 K_{2}+\bar{K}_{2}$, or $C_{4}+\bar{K}_{2}$.

Case 1. $H=2 K_{3}$.

If $G=H \cup K_{1}$, it is easily verified that $\psi=\bar{\psi}=3$. Now we may assume that $G \supset H \cup K_{1}$ properly. Then there is a point $v_{i}$ in $G$ which is adjacent to $w$. Without loss of generality we may assume that $w A v_{i}$. On the other hand, there is at least one point $v_{i}, i=4,5$ or 6 , which is not adjacent to $w$, say $v_{4}$ as shown in Figure 3, otherwise all three points $v_{i}$, $i=4,5$, and 6 are adjacent to $w$ and so $\left\{v_{4}, v_{5}, v_{6}, w\right\}$ induces $K_{4}$, a contradiction. 


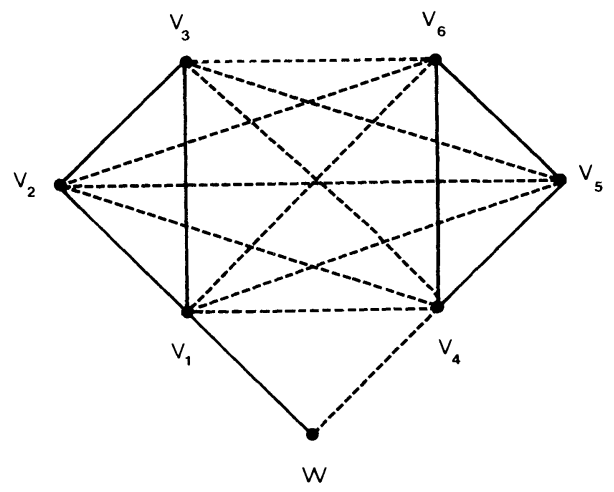

Figure 3. A step in the proof of Case 1

Then it is easy to see that $\psi(G)=4$ regardless of whether or not $w A v_{i}$ for $i=2,3,5,6$, a contradiction.

Case 2. $H=2 K_{2}+\bar{K}_{2}$.

As $\psi=\bar{\psi}=3$, we know that $\chi, \bar{\chi} \leq 3$ so by Lemma 2 c, $\chi=\bar{\chi}=3$. Thus by Corollary $2 \mathrm{~d}, \bar{G}$ contains a triangle. As $H=2 K_{2}+\bar{K}_{2}=G-w$, it follows that $G$ contains $C_{4} \cup K_{2}$ as an induced subgraph. Hence there are two possibilities: either $\bar{G} \supset F_{1}$ or $\bar{G} \supset F_{2}$, where $F_{1}, F_{2}$ are the graphs illustrated in Figure 4, which we now consider as two subcases.
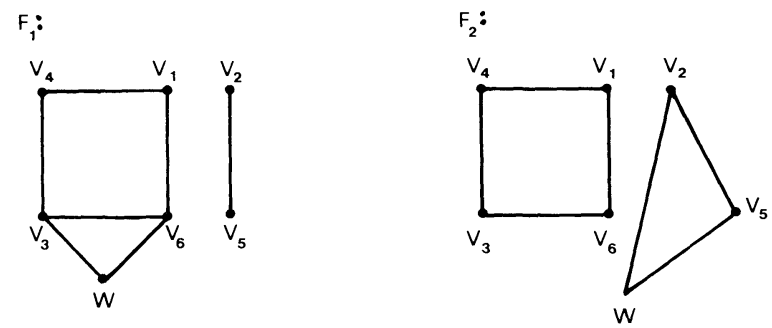

Figure 4. A step in the proof of Case 2

Case 2a. $\bar{G} \supset F_{1}$.

If $\bar{G} \neq F_{1}$, then $w$ is adjacent to at least one more point of $G$, i.e., to $v_{1}, v_{2}, v_{4}$, or $v_{5}$. We may assume that $w$ is adjacent to $v_{1}$ or $v_{2}$ from the symmetry of $F_{1}$. In either case, $\bar{\psi}=4$, a contradiction. On the other hand, if $\bar{G}=F_{1}$ then $\bar{\psi}=4$, a contradiction.

Case 2b. $\bar{G} \supset F_{2}$.

If $\bar{G}=F_{2}$, then $\psi=\bar{\psi}=3$. If $\bar{G} \neq F_{2}$, then $w$ is adjacent to one of the points $v_{i}, i=1,3,4$ or 6 . From the symmetry of $F_{2}$, we may assume that $w A v_{1}$. Then it is easy to see that $\psi=4$, a contradiction. 
Case 3. $H=C_{4}+\bar{K}_{2}$.

Since $\bar{G} \supset K_{3}$ from Corollary $2 \mathrm{~d}$, and $\bar{H}=3 K_{2}$, it follows that $\bar{G} \supset 2 K_{2} \cup K_{3}$. We may assume without loss of generality that $\left\{v_{2}, v_{5}, w\right\}$ induces $K_{3}$ in $\bar{G}$; see Figure 5. If $\bar{G}=2 K_{2} \cup K_{3}$, then $\psi=\bar{\psi}=3$. If $\bar{G} \neq 2 K_{2} \cup K_{3}$, then $w$ must be adjacent to at least one of $v_{i}, i=1,3,4$ or 6. Assuming now that $w A v_{1}$, we see that $\bar{\psi}=4$, a contradiction.

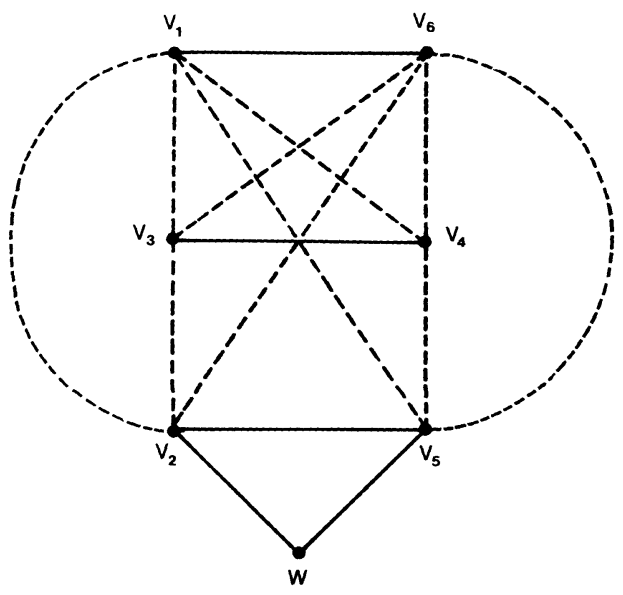

Figure 5. A step in the proof of Case 3

LEMMA 4c. If $G$ is a graph of order 7 such that neither $G$ nor $\bar{G}$ contains two point-disjoint triangles, then $\psi$ or $\bar{\psi}$ is at least 4.

Proof. Assume that $\psi=\bar{\psi}=3$, then $\chi, \bar{\chi} \leq 3$ since $\chi \leq \psi$. By applying Lemma $2 \mathrm{c}, \chi=\bar{\chi}=3$. Thus $G \supset K_{3} \cup 2 K_{2}$ or $G \supset 2 K_{3} \cup K_{1}$ by Corollary $2 \mathrm{~d}$. But by the hypothesis, $G$ cannot contain two point-disjoint triangles and so, $G, \bar{G} \supset K_{3} \cup 2 K_{2}$. Now we label the points of $K_{3} \cup 2 K_{2}$ as in Figure 6.

G:

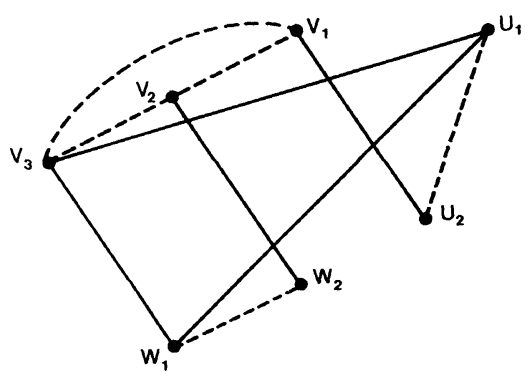

FIGURE 6. A labelling of $K_{3} \cup 2 K_{2}$ 
By the symmetry of $G$ and $\bar{G}$, it is sufficient to handle only the case $u_{2} A w_{2}$. By the hypothesis that $G$ cannot contain two point-disjoint triangles, $v_{1} A w_{2}$ and $v_{2} A u_{2}$. Then regardless of the presence or absence of other lines, we can easily verify that $\bar{\psi}=4$, a contradiction.

LEMMA 4d. There are no graphs of order at least 8 such that $\psi=\bar{\psi}=3$.

Proof. Assume that $G$ has order 8 and $\psi=\bar{\psi}=3$. Then $\chi=\bar{\chi}=3$ by Lemma 2c. Thus both $G$ and $\bar{G}$ contain $2 K_{3} \cup K_{2}$ as a spanning subgraph by Corollary $2 \mathrm{~d}$. The subgraph of $G$ induced by the set of points of $2 K_{3}$ must be one of the three graphs, $2 K_{3}, 2 K_{2}+\bar{K}_{2}$ or $C_{4}+\bar{K}_{2}$ of Lemma 4a. We now divide the proof into three cases:

Case 1. $G$ contains $2 K_{3}$ as an induced subgraph.

By Corollary 2 d, both $G$ and $\bar{G}$ contain $2 K_{3} \cup K_{2}$ hence of course $\bar{G} \supset 2 K_{3}$. It is convenient to label $\bar{G}$ as in Figure 7 .

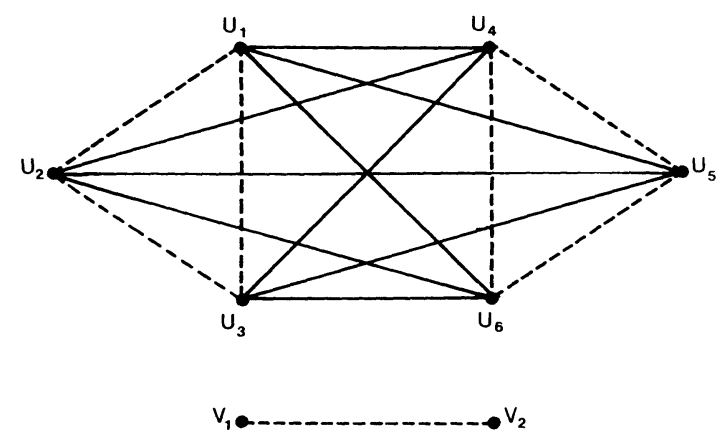

FIGURE 7. A subgraph of $\bar{G}$

By symmetry, we may assume that both point sets $\left\{u_{3}, u_{6}, v_{1}\right\}$ and $\left\{u_{2}, u_{5}, v_{2}\right\}$ induce $K_{3}$ in $\bar{G}$. Then it is easily verified that $\bar{\psi}=4$.

Case 2. $G$ contains $2 K_{2}+\bar{K}_{2}$ as an induced subgraph.

Let $F_{1}, F_{2}$ be the graphs illustrated in Figure 8. 
$F_{1}:$

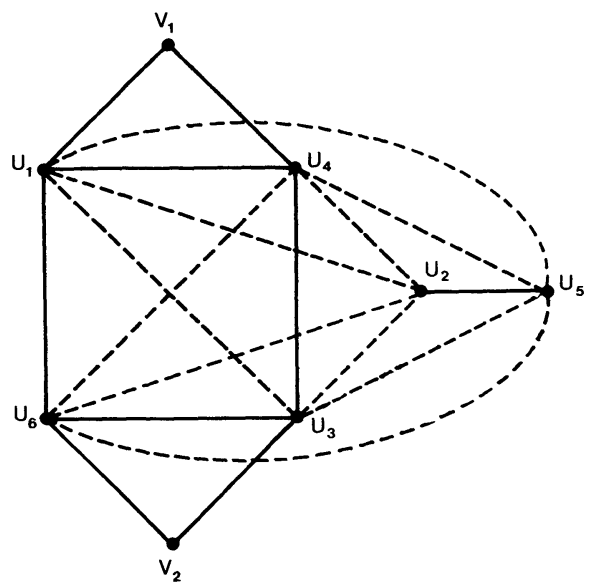

$F_{2}:$

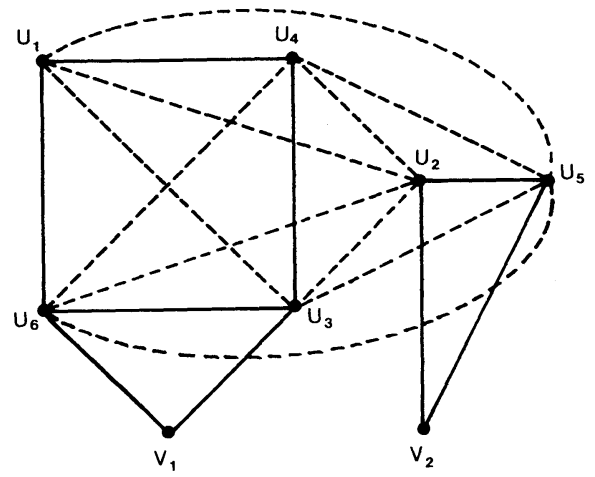

FIGURE 8. Subgraphs $F_{1}$ and $F_{2}$ of $\bar{G}$

Since $\bar{G} \supset 2 K_{3}$ by Corollary $2 \mathrm{~d}$, there are two possibilities: either $\bar{G} \supset F_{1}$ or $\bar{G} \supset F_{2}$. However in either case, $\bar{\psi}=4$.

Case 3. $G$ contains $C_{4}+\bar{K}_{2}$ as an induced subgraph.

Since $\bar{G} \supset 2 K_{3}$ by Corollary $2 \mathrm{~d}$, we may assume that both $\left\{v_{1}, u_{2}, u_{5}\right\}$ and $\left\{v_{2}, u_{3}, u_{4}\right\}$ induce $K_{3}$ in $\bar{G}$, see Figure 9 , and thus $\bar{\psi}=4$, a contradiction.

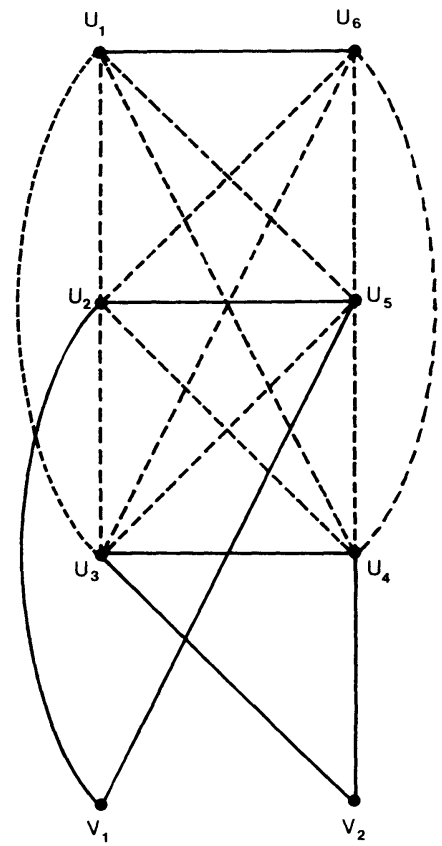

FIGURE 9. A subgraph of $\bar{G}$ 
Combining the preceding four lemmas, we obtain the following result.

LEMMA 4e. Let $G$ be a graph of order at least 7 , then $G$ has $\psi=\bar{\psi}=3$ if and only if $G$ is one of the six graphs, $2 K_{3} \cup K_{1}, K(3,3,1), C_{4} \cup C_{3}$, $2 K_{2}+\bar{K}_{3}, 2 K_{2} \cup K_{3}$ and $K(3,2,2)$.

We are now ready to specify all the graphs with $\psi=\bar{\psi}=3$.

THEOREM 4. There are exactly 41 graphs $G$ such that both $G$ and $\bar{G}$ have achromatic number 3: six have order 7, twenty are of order 6, fourteen of order 5 and just one of order 4.

Proof. By Lemma 4d, we know that there are no such graphs of order $p \geq 8$. Lemma 4e lists all six graphs with $p=7$ and Figure 2 shows them. To complete the list of all the graphs with $\psi=\bar{\psi}=3$, we had to resort to the method of brute force by an exhaustive inspection of Appendix I of [1] for $p=4,5$, and 6 .

As the determination of all graphs with $\psi=\bar{\psi}=n \geq 4$ appears to be hopelessly complicated, we can realistically ask only for the construction of additional families of graphs with $\psi=\bar{\psi}$.

\section{REFERENCES}

1. F. Harary, Graph Theory, Addison-Wesley, Reading (1969).

2. F. Harary, S. T. Hedetniemi, and G. Prins, An interpolation theorem for graphical homomorphisms, Port. Math., 26 (1967), 453-462.

3. E. A. Nordhaus and J. W. Gaddum, On complimentary graphs, Amer. Math. Monthly, 63 (1956), 175-177.

Received February 18, 1980.

NIPPON IKA UNIVERSITY

KAWASAKI, 211, JAPAN

UNIVERSITY OF MICHIGAN

ANN ARBOR, MI 48109

AND

UNIVERISITY OF CALIFORNIA

SANTA BARBARA, CA 93106 



\section{PACIFIC JOURNAL OF MATHEMATICS \\ EDITORS}

DONALD BABBITT (Managing Editor)

University of California

Los Angeles, CA 90024

\section{Hugo Rossi}

University of Utah

Salt Lake City, UT 84112

C. C. Moore and Arthur Ogus

University of California

Berkeley, CA 94720
J. DugundiI

Department of Mathematics

University of Southern California

Los Angeles, CA 90089-1113

R. FINN and H. SAMELSON

Stanford University

Stanford, CA 94305

ASSOCIATE EDITORS
R. ARens
E. F. BECKENBACH
B. H. NeumanN
F. WOLF
K. YoshidA (1906-1982)

\section{SUPPORTING INSTITUTIONS}

UNIVERSITY OF ARIZONA

UNIVERSITY OF BRITISH COLUMBIA

CALIFORNIA INSTITUTE OF TECHNOLOGY

UNIVERSITY OF CALIFORNIA

MONTANA STATE UNIVERSITY

UNIVERSITY OF NEVADA, RENO

NEW MEXICO STATE UNIVERSITY

OREGON STATE UNIVERSITY
UNIVERSITY OF OREGON

UNIVERSITY OF SOUTHERN CALIFORNIA

STANFORD UNIVERSITY

UNIVERSITY OF HAWAII

UNIVERSITY OF TOKYO

UNIVERSITY OF UTAH

WASHINGTON STATE UNIVERSITY

UNIVERSITY OF WASHINGTON 


\section{Pacific Journal of Mathematics}

Vol. 104, No. 1

May, 1983

Nestor Edgardo Aguilera and Eleonor Ofelia Harboure de Aguilera, On

the search for weighted norm inequalities for the Fourier transform $\ldots \ldots .1$

Jin Akiyama, Frank Harary and Phillip Arthur Ostrand, A graph and its complement with specified properties. VI. Chromatic and achromatic numbers ......................................... 15

Bing Ren Li, The perturbation theory for linear operators of discrete type . . . 29

Peter Botta, Stephen J. Pierce and William E. Watkins, Linear

transformations that preserve the nilpotent matrices .............. 39

Frederick Ronald Cohen, Ralph Cohen, Nicholas J. Kuhn and Joseph

Alvin Neisendorfer, Bundles over configuration spaces .......... 47

Luther Bush Fuller, Trees and proto-metrizable spaces . . . . . . . . . . 55

Giovanni P. Galdi and Salvatore Rionero, On the best conditions on the

gradient of pressure for uniqueness of viscous flows in the whole space . . 77

John R. Graef, Limit circle type results for sublinear equations $\ldots \ldots \ldots \ldots 85$

Andrzej Granas, Ronald Bernard Guenther and John Walter Lee,

Topological transversality. II. Applications to the Neumann problem for

$y^{\prime \prime}=f\left(t, y, y^{\prime}\right) \ldots \ldots \ldots \ldots \ldots \ldots \ldots \ldots \ldots \ldots \ldots \ldots \ldots \ldots \ldots . \ldots 5$

Richard Howard Hudson and Kenneth S. Williams, Extensions of

theorems of Cunningham-Aigner and Hasse-Evans . . . . . . . . . . . 111

John Francis Kurtzke, Jr., Centralizers of irregular elements in reductive algebraic groups

James F. Lawrence, Lopsided sets and orthant-intersection by convex

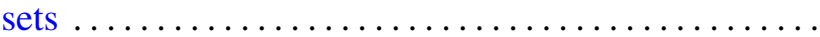

Åsvald Lima, G. H. Olsen and U. Uttersrud, Intersections of $M$-ideals and

$G$-spaces

Wallace Smith Martindale, III and C. Robert Miers, On the iterates of derivations of prime rings

Thomas H. Pate, Jr, A characterization of a Neuberger type iteration procedure that leads to solutions of classical boundary value problems

Carl L. Prather and Ken Shaw, Zeros of successive iterates of multiplier-sequence operators

Billy E. Rhoades, The fine spectra for weighted mean operators

Rudolf J. Taschner, A general version of van der Corput's difference theorem

Johannes A. Van Casteren, Operators similar to unitary or selfadjoint ones 